\title{
Author Correction: Chromatin-associated RNAs as facilitators of functional genomic interactions
}

\section{Xiao Li(D) and Xiang-Dong Fu(B)}

Nature Reviews Genetics (2019) https://doi.org/10.1038/s41576-019-0135-1

Published online 03 June 2019

In figures 1, 4 and 5 of this article there were several instances where the transcribing RNA polymerase II (and the RNA-DNA hybrid inside it) had the incorrect left-right orientation. These figures have now been corrected. Additionally, minor text errors in the main text have been corrected: in the section "Nascent RNAs in gene regulation", the phrase "histone shock gene" has been corrected to "heat shock gene", and "chromatin spreading" has been updated specifically to "heterochromatin spreading" when discussing the functions of Xist-interacting proteins.

https://doi.org/10.1038/s41576-019-0155-x I Published online 4 July 2019

\section{Author Correction: Genomics of disease risk in globally diverse populations}

\section{Deepti Gurdasani(i), Inês Barroso (1), Eleftheria Zeggini and Manjinder S. Sandhu (1)}

Nature Reviews Genetics (2019) https://doi.org/10.1038/s41576-019-0144-0

Published online 24 June 2019

In the originally published article, the following acknowledgement was missing: D.G. is funded by a UKRI HDR-UK Innovation Fellowship (reference number MR/S003711/1). The article has now been corrected online.

https://doi.org/10.1038/s41576-019-0153-z I Published online 3 July 2019

\section{Author Correction: Challenges in measuring and understanding biological noise}

\section{Nils Eling (D), Michael D. Morgan (D) and John C. Marioni(iD)}

Nature Reviews Genetics (2019) https://doi.org/10.1038/s41576-019-0130-6

Published online 21 May 2019

In the reference list of the originally published article, the reference annotation "This study used an scRNA-seq and matched smFISH approach to model the variability versus mean expression relationship while accounting for technical noise" should have been associated with reference 56 (Grün, Kester \& van Oudenaarden, Nat. Methods (2014)) rather than with reference 57. The article has now been corrected online.

https://doi.org/10.1038/s41576-019-0142-2 I Published online 3 June 2019 